\title{
Anakinra after treatment with corticosteroids alone or with tocilizumab in patients with severe COVID-19 pneumonia and moderate hyperinflammation. A retrospective cohort study
}

\author{
Ismael Francisco Aomar-Millán ${ }^{1}$ (1) - Juan Salvatierra ${ }^{2}$. Úrsula Torres-Parejo ${ }^{3}$ Naya Faro-Miguez ${ }^{4}$. \\ José Luis Callejas-Rubio ${ }^{1}$. Ángel Ceballos-Torres ${ }^{1} \cdot$ María Teresa Cruces-Moreno $^{5}$. \\ Francisco Javier Gómez-Jiménez ${ }^{1}$. José Hernández-Quero ${ }^{4}$. Francisco Anguita-Santos ${ }^{4}$
}

Received: 16 September 2020 / Accepted: 2 December 2020 / Published online: 5 January 2021

(c) Springer Nature Switzerland AG part of Springer Nature 2021

\begin{abstract}
Introduction Little evidence appears to exist for the use of anakinra, a recombinant interleukin-1 receptor antagonist, after non-response to treatment with corticosteroids alone or combined with tocilizumab in patients with severe COVID-19 pneumonia and moderate hyperinflammatory state.

Patients and Methods A retrospective observational cohort study was carried out involving 143 patients with severe COVID19 pneumonia and moderate hyperinflammation. They received standard therapy along with pulses of methylprednisolone (group 1) or methylprednisolone plus tocilizumab (group 2), with the possibility of receiving anakinra (group 3) according to protocol. The aim of this study was to assess the role of anakinra in the clinical course (death, admission to the intensive care ward) during the first 60 days after the first corticosteroid pulse. Clinical, laboratory, and imaging characteristics as well as infectious complications were also analyzed.

Results 74 patients $(51.7 \%)$ in group 1, $59(41.3 \%)$ patients in group 2, and 10 patients (7\%) in group 3 were included. 8 patients $(10.8 \%)$ in group 1 died, $6(10.2 \%)$ in group 2, and $0(0 \%)$ in group 3. After adjustment for age and clinical severity indices, treatment with anakinra was associated with a reduced risk of mortality (adjusted hazard ratio $0.518,95 \%$ CI $0.265-0.910 ; p=0.0437)$. Patients in group 3 had a lower mean CD4 count after 3 days of treatment. No patients in this group presented infectious complications.

Conclusions In patients with moderate hyperinflammatory state associated with severe COVID-19 pneumonia, treatment with anakinra after non-response to corticosteroids or corticosteroids plus tocilizumab therapy may be an option for the management of these patients and may improve their prognosis.
\end{abstract}

Keywords Anakinra $\cdot$ Tocilizumab $\cdot$ Methylprednisolone $\cdot$ Hyperinflammation $\cdot$ Cytokine storm syndrome $\cdot$ COVID-19

Ismael Francisco Aomar-Millán, Juan Salvatierra, Úrsula TorresParejo and Francisco Anguita-Santos have contributed equally to the work.

Ismael Francisco Aomar-Millán

iaomarmillan@hotmail.com

1 Department of Internal Medicine, San Cecilio University

Hospital, Hospital Universitario San Cecilio, Avda. del

Conocimiento s/n, 18016 Granada, Spain

2 Department of Rheumatology, San Cecilio University Hospital, Granada, Spain

\author{
Abbreviations \\ SARS-CoV-2 Severe acute respiratory syndrome \\ coronavirus- 2 \\ COVID-19 Human coronavirus disease caused by \\ SARS-CoV-2 \\ IL Interleukin
}

3 Department of Statistics and Operational Research, University of Granada, Granada, Spain

4 Department of Infectious Diseases, San Cecilio University Hospital, Granada, Spain

5 Department of Intensive Care Unit, San Cecilio University Hospital, Granada, Spain 


$\begin{array}{ll}\text { TNF- } \alpha & \text { Tumor necrosis factor alpha } \\ \text { ARDS } & \text { Adult respiratory distress syndrome } \\ \text { MAS } & \text { Macrophage activation syndrome } \\ \text { CT } & \text { Computed tomography } \\ \text { MTP } & \text { Methylprednisolone } \\ \text { CTS } & \text { Corticosteroids } \\ \text { TCZ } & \text { Tocilizumab } \\ \text { ICU } & \text { Intensive care unit } \\ \text { COPD } & \text { Chronic obstructive pulmonary disease } \\ \text { qSOFA } & \text { Quick Sepsis-related Organ Failure } \\ & \text { Assessment } \\ \text { ICU } & \text { Intensive care unit } \\ \text { GM-CSF } & \text { Granulocyte-macrophage colony-stimu- } \\ & \text { lating factor }\end{array}$

\section{Introduction}

In December 2019, a severe respiratory syndrome associated with pneumonia caused by a new human coronavirus called severe acute respiratory syndrome coronavirus-2 (SARSCoV-2) was identified and spread rapidly to become a global public health problem [1]. The syndrome associated with this infection presents several clinical manifestations with a direct correlation between the severity of pneumonia, systemic inflammation, progression to respiratory failure, and death [2].

The clinical course of COVID-19 is characterized by three different phases [3]. In the initial phase, there is strong viral replication accompanied by influenza-like symptoms. The second phase is usually associated with high fever and pneumonia-like symptoms, despite a steady decrease in viremia. Subsequently, some patients develop a third hyperinflammatory phase with an increase in cytokine storm markers such as C-reactive protein, ferritin, and D-dimers [4] along with a significant decrease in T lymphocytes.

Cytokine storm is the result of an imbalance between antiviral and proinflammatory responses in which cytokines such as IL-1, IL-6, IL-18, and tumor necrosis factor alpha $(\mathrm{TNF}-\alpha)$ are released, causing lung damage and probably predisposing patients to thromboembolic events. Based on the high levels of these proinflammatory cytokines, several drugs are being used to block them and control this type of hyperinflammation.

Adult respiratory distress syndrome (ARDS) as a result of hyperinflammation is considered the leading cause of death in these patients $[5,6]$. Once the hyperinflammatory state develops, prompt, individualized treatment is essential to control it and avoid multiorgan failure and death.

In patients with severe SARS-CoV-2 infection, increased IL-1 alpha and beta expression have been demonstrated prior to the deterioration in respiratory function, which supports the involvement of IL-1 in the physiopathology of ARDS in these patients [7].

Anakinra is a recombinant $\mathrm{IL}-1$ receptor antagonist that is commonly used to treat hyperinflammatory conditions such as macrophage activation syndrome (MAS). Anakinra was shown to increase the survival rate in patients with sepsis who meet the criteria for MAS, and who had liver dysfunction and disseminated intravascular coagulation [8]. This drug has also been used to treat several rare syndromes characterized by hyperinflammation mediated by the altered regulation of cytokine responses [9, 10]. Several studies have reported the benefits of anakinra as an initial treatment for patients with hyperinflammation associated with severe SARS CoV-2 infection [11-14], although these studies did not focus on patients who failed to respond to corticosteroid pulses or tocilizumab therapy alone or in combination.

In the present study, we aimed to analyze the usefulness of IL-1 blockade with anakinra in a series of patients with severe pneumonia and moderate hyperinflammation after failure to respond to corticosteroid alone or with tocilizumab therapy. We also aimed to identify laboratory and clinical factors predictive of the response to anakinra.

\section{Material and methods}

\section{Patients and study design}

This retrospective cohort study included 143 patients admitted consecutively to San Cecilio University Hospital of Granada, Spain between 15 March and 15 May 2020 with severe SARS-CoV-2 pneumonia and hyperinflammation. The diagnosis was confirmed by PCR in a nasopharyngeal swab, and all patients had fever $>38{ }^{\circ} \mathrm{C}$ for at least 5 days since the onset of symptoms, and met two of the following laboratory criteria: PCR > $90 \mathrm{mg} / \mathrm{L}$, ferritin level > $500 \mu \mathrm{g} / \mathrm{L}$, D-dimer level $>0.5 \mathrm{mg} / \mathrm{L}$. Severe pneumonia was considered present when basal oxygen saturation was $<93 \%$ or partial $\mathrm{O}_{2}$ pressure was $<65 \mathrm{mmHg}$, with radiological evidence (chest X-ray or chest CT scan) of unilobar or multilobar involvement compatible with COVID-19 influenza [15].

All patients received standard treatment according to our hospital protocol, except when there were contraindications for electrocardiographic studies. The protocol consisted of hydroxychloroquine $(800 \mathrm{mg} /$ day on the first day and $400 \mathrm{mg} /$ day for another 4 days), azithromycin (500 mg on the first day and $250 \mathrm{mg} /$ day for 5 days), lopinavir/ritonavir (800/200 mg daily for 14 days), and ceftriaxone ( $2 \mathrm{~g}$ per day for 7-10 days) together with thromboembolism prophylaxis with bemiparin at a dose adjusted according to thrombotic risk (low risk $3500 \mathrm{UI} /$ day, intermediate risk 5000 IU/day).

The immunosuppressive treatment protocol our hospital recommended for patients with hyperinflammation 
associated with severe SARS-CoV-2 pneumonia consisted of three phases. (1) Intravenous methylprednisolone (MTP) $2 \mathrm{mg} / \mathrm{kg} /$ day was given for 3 days, with the possibility of 2 further days if necessary due to partial clinical improvement (group 1, corticosteroid treatment). (2) After evaluating the quality of life and life expectancy for each patient prior to admission, the medical team could opt to use tocilizumab for those patients who did not show clinical improvement or whose respiratory condition worsened within $48 \mathrm{~h}$ (group 2, CTS + TCZ). Tocilizumab was administered as a single intravenous dose adjusted according to body weight: patients $<75 \mathrm{~kg}$ received $400 \mathrm{mg}$ iv; those $>75 \mathrm{~kg}$ received $600 \mathrm{mg}$. (3) In patients whose respiratory condition worsened (defined as requiring increased oxygen) within $48 \mathrm{~h}$ after starting treatment with TCZ, subcutaneous anakinra was administered. According to our center's protocol, anakinra could be administered in addition to tocilizumab in patients with serum levels of IL- $6<40 \mathrm{pg} / \mathrm{mL}$ if there was no respiratory improvement $48 \mathrm{~h}$ after receiving MTP boluses (group 3). The dose of anakinra was adjusted according to body weight. On the first day, patients who weighed $50-60 \mathrm{~kg}$ received $100 \mathrm{mg} / 12 \mathrm{~h}$, patients who weighted $60-75 \mathrm{~kg}$ received $100 \mathrm{mg} / 8 \mathrm{~h}$, and patients who weighed $>75 \mathrm{~kg}$ received $100 \mathrm{mg} / 6 \mathrm{~h}$. On the second day, all patients received $100 \mathrm{mg} / 12 \mathrm{~h}$ from day 2 to day 6 .

On admission, demographic characteristics (age, gender) and comorbidities (hypertension, diabetes, ischemic heart disease, kidney failure, heart failure, obesity, chronic obstructive pulmonary disease (COPD), asthma, obstructive sleep apnea) were recorded, along with laboratory data, clinical severity indices (CURB-65 and Quick Sepsis-related Organ Failure Assessment [qSOFA]), use of antiaggregants, anticoagulants, and ACEI/ARA-2, and radiological severity. The CURB-65 score is a quantitative measure of pneumonia severity [16], and the qSOFA score identifies patients with infection who are at high risk of death [17].

The following laboratory parameters were recorded before and $72 \mathrm{~h}$ after treatment: C-reactive protein, serum ferritin, total lymphocyte count with CD4 and CD8 subpopulations, and D-dimers. The levels of CRP and ferritin were also analyzed 1 month after the first MTP bolus.

The severity of unilobar or multilobar pulmonary involvement was evaluated by chest X-ray and CT scan. A semiquantitative scoring system developed by the British Thoracic Imaging Society [18] was used to estimate lung involvement based on the affected area, and involvement was classified as mild $(<25 \%)$, moderate $(25-50 \%)$, or severe $(>51 \%)$.

The primary outcomes of interest were the numbers of deaths and intensive care unit (ICU) admissions within 60 days after receiving the first corticosteroid pulse. Data were obtained from the digital medical records managed by our regional healthcare service and were analyzed with
SPSS software (version 24.1 licensed to the University of Granada). The study protocol was implemented in accordance with the principles of the Declaration of Helsinki. Each patient or their legal representative or closest relative was informed about the use of off-label treatments, and written informed consent was obtained to use these treatments, and to analyze and process the data. The study was approved by the Granada provincial research ethics committee (Comite Etico de Investigación Provincial de Granada).

\section{Results}

A total of 143 patients admitted consecutively between 15 March and 15 May 2020 with severe SARS-CoV-2 pneumonia who met predefined hyperinflammation criteria were included in this study. For analysis and comparison, the patients were divided into three groups: $74(51.7 \%)$ in group 1 (CTS), $59(41.3 \%)$ in group 2 (CTS + TCZ), and $10(7 \%)$ in group 3 (CTS with or without TCZ + anakinra). Table 1 shows the clinical and demographic characteristics and laboratory abnormalities in all patients treated with CTS, CTS + TCZ, or CTS with or without TCZ + anakinra upon admission, and their clinical course.

In the entire cohort, $52 \%$ of the patients were older than 65 years. Regarding the frequencies of comorbidities, $52 \%$ had hypertension, $41 \%$ had obesity, $25 \%$ had respiratory diseases, $18 \%$ had diabetes, $11 \%$ had kidney failure, $9 \%$ had heart failure, and 6\% had ischemic heart disease. More than 2 comorbidities were recorded for 49 (31\%) patients. No differences were found in the percentage frequencies of comorbidities between the different treatment groups.

On admission, chest X-ray revealed multilobar infiltrates in 109 patients (76\%) and chest CT scan showed moderate to severe involvement in 65 (46\%) patients.

No differences on admission were found between the three treatment groups regarding demographic characteristics, comorbidities, CURB-65 or qSOFA score, or degree of radiological involvement (Table 2).

Fifty-two percent of the patients received treatment with corticosteroid pulses only (group 1), $41 \%$ received corticosteroids plus tocilizumab (group 2), and 7\% received anakinra (group 3) after failure to respond to treatment with corticosteroids alone or with tocilizumab.

Anakinra was used in 4 patients after corticosteroids and in 6 patients after corticosteroids plus tocilizumab. Regarding the use of anakinra as second-line therapy due to low IL-6 levels, only 1 patient who had previously received corticosteroids received anakinra due to a serum level of IL6 $<40 \mathrm{pg} / \mathrm{mL}$. Although the recommended protocol contemplated the possibility of administering anakinra after corticosteroids in patients with serum levels of IL- $6<40 \mathrm{pg}$ / $\mathrm{mL}$, the medical team responsible for the patients decided 
Table 1 Clinical and demographic characteristics on admission, laboratory abnormalities, and clinical course in patients treated with CTS, CTS + TCZ or anakinra

\begin{tabular}{|c|c|c|c|c|}
\hline & CTS (Group 1) & CTS + TCZ (Group 2) & CTS w/wo TCZ + anakinra (Group 3) & p-value* \\
\hline Age, mean (SD), years & $68.18(13.68)$ & $62.17(11.45)$ & $60.80(11.43)$ & 0.950 \\
\hline Male sex, n (\%) & $39(52.7)$ & $40(67.8)$ & $10(100)$ & 0.011 \\
\hline CURB-65 score, mean (SD) & $1.81(0.84)$ & $1.80(0.69)$ & $1.50(0.53)$ & 0.828 \\
\hline qSOFA score, mean (SD) & $0.55(0.67)$ & $0.80(0.78)$ & $0.40(0.52)$ & 0.680 \\
\hline \multicolumn{5}{|l|}{ Comorbidities n (\%): } \\
\hline -Arterial hypertension & $37(50)$ & $32(54.2)$ & $4(40)$ & 0.469 \\
\hline -COPD, asthma & $16(21.6)$ & $18(30.5)$ & $2(20)$ & 0.696 \\
\hline -Obesity & $32(43.2)$ & $24(40.7)$ & $3(30)$ & 0.453 \\
\hline -Ischemic heart disease & $4(5.4)$ & $4(6.8)$ & $0(0)$ & 0.425 \\
\hline -Heart insufficiency & $8(10.8)$ & $5(8.5)$ & $0(0)$ & 0.300 \\
\hline -Diabetes & $12(16.2)$ & $12(20.3)$ & $2(20)$ & 0.877 \\
\hline -Renal insufficiency & $11(14.9)$ & $4(6.8)$ & $0(0)$ & 0.262 \\
\hline Intubated, n (\%) & $2(2.7)$ & $7(11.9)$ & $2(20)$ & 0.130 \\
\hline Intubation duration, median (IQR), days & $29.50(13)$ & $29(31.5)$ & $24.50(25)$ & 0.272 \\
\hline $\begin{array}{l}\text { ICU admission duration median (IQR), } \\
\text { days }\end{array}$ & $36(4)$ & $14.5(24)$ & $30.50(25)$ & 0.374 \\
\hline Death, $n(\%)$ & $8(10,8)$ & $6(10,2 \%)$ & $0(0 \%)$ & 0.280 \\
\hline CRP (mg/L), mean (median; SD) & $99.38(78.00 ; 78.45)$ & $\begin{array}{l}136.69 \\
(112.60 ; 72.60)\end{array}$ & $\begin{array}{l}118.54 \\
(94.10 ; 90.25)\end{array}$ & 0.501 \\
\hline $\begin{array}{l}\text { Procalcitonin }(\mathrm{ng} / \mathrm{mL}) \text {, mean (median; } \\
\text { SD) }\end{array}$ & $\begin{array}{l}0.19 \\
(0.08 ; 0.32)\end{array}$ & $\begin{array}{l}0.28 \\
(0.16 ; 0.42)\end{array}$ & $\begin{array}{l}0.17 \\
(0.11 ; 0.16)\end{array}$ & 0.933 \\
\hline Lymphopenia $(<600$ cells/uL) $n(\%)$ & $62(83.8)$ & $46(78.0)$ & $8(80)$ & 0.925 \\
\hline CD4 (cells/uL), mean (median; SD) & $418.26(392.00 ; 212.59)$ & $440.54(429.50 ; 210.85)$ & $360.00(343.50 ; 265.02)$ & 0.237 \\
\hline CD8 (cells/uL), mean (median; SD) & $216.46(183.00 ; 147.75)$ & $256.47(206.00 ; 207.24)$ & $395.50(424.00 ; 229.70)$ & 0.589 \\
\hline Ferritin (ng/mL), mean (median; SD) & $846.86(676.00 ; 637.26)$ & $1184.30(915.20 ; 768.62)$ & $1032.88(1129.20 ; 517.59)$ & 0.497 \\
\hline IL-6 > $40(\mathrm{pg} / \mathrm{mL}), n(\%)$ & $73(98.6)$ & $22(37.3)$ & $9(90)$ & 0.203 \\
\hline D-Dimer (mg/L), mean (median;SD) & $6.42(0.99 ; 38.59)$ & $5.31(1.21 ; 16.08)$ & $1.11(0.47 ; 2.06)$ & 0.669 \\
\hline \multicolumn{5}{|l|}{$\begin{array}{l}\text { Antiaggregants, Anticoagulants } \\
\text { AARS blockers, } n(\%) \text { : }\end{array}$} \\
\hline -Antiaggregants & $15(20.3)$ & 7 (11.9) & $0(0)$ & 0.162 \\
\hline -Anticoagulants & $6(8,1)$ & $3(5.1)$ & $0(0)$ & 0.395 \\
\hline -ACEI/ARA-2 & $33(44.6)$ & $27(45.8)$ & $4(40)$ & 0.754 \\
\hline
\end{tabular}

*For comparisons of group 1 and group 2 versus group 3

CTS corticosteroids, TCZ, tocilizumab, IQR interquartile range, $S D$ standard deviation, $q S O F A$ Quick Sepsis-related Organ Failure Assessment; AARS, angiotensin aldosterone renin system; ACEI, angiotensin-converting enzyme inhibitors; ARA-2, angiotensin receptor antagonist 2; CRP, C-reactive protein; COPD: chronic obstructive pulmonary disease

to use anakinra in 3 patients after corticosteroids in despite their serum level of IL6 $>40 \mathrm{pg} / \mathrm{mL}$ because 2 patients had a history of diverticulitis, and 1 patient suffered from abdominal pain.

All 14 deaths (9.8\%) observed during the 60 day followup period after admission occurred within the first 30 days: $8(10.8 \%)$ in group $1,6(10.2 \%)$ in group 2 , and no deaths during this period in group 3. After adjustment for age and clinical severity indices, treatment with anakinra was associated with a reduced risk of mortality (adjusted hazard ratio $0.518,95 \%$ CI $0.265-0.910 ; p=0.0437)$. Of the 10 patients who received anakinra, $4(40 \%)$ had previously received only corticosteroid pulses, and $6(60 \%)$ had received combined therapy with corticosteroids and tocilizumab. More patients in group $2(7,11.9 \%)$ and group $3(2,20 \%)$ were intubated in comparison to group $1(2,2.7 \%)$, with a mean duration of UCI stay of around 4 weeks. In our study, deaths in group 1 (corticosteroids) occurred after an average of 8.63 days [median $(\mathrm{IQR})=8(7-10)]$ and deaths in group 2 (corticosteroids plus tocilizumab) occurred after an average of 22 days [median $(I Q R)=15(6-38)]$. These numbers suggest that we can rule out the likelihood that patients whose clinical course evolved rapidly would die by day 3 , and so remained in these groups. By day 60 after admission, none of the patients was in the ICU, 
Table 2 Analysis of the association between treatment and patient characteristics

\begin{tabular}{lllll}
\hline Factors & Variables & Chi-squared test & $\begin{array}{l}\text { Degrees of } \\
\text { freedom }\end{array}$ & p-value \\
\hline Comorbidities & Arterial hypertension & 1.654 & 2 & 0.437 \\
& COPD, asthma & 1.529 & 2 & 0.466 \\
& Obesity & 0.651 & 2 & 0.722 \\
& Ischemic heart disease & 0.755 & 2 & 0.686 \\
& Heart failure & 1.292 & 2 & 0.524 \\
& Diabetes & 0.399 & 2 & 0.819 \\
Score & Kidney failure & 3.546 & 2 & 0.170 \\
& & & & \\
& CURB-65 & 4.878 & 6 & 0.899 \\
Plain chest X-ray & qSOFA & 6.991 & 4 & 0.322 \\
CT involvement & Clain chest X-ray & 3.112 & 4 & 0.539 \\
\hline
\end{tabular}

qSOFA Quick Sepsis-related Organ Failure Assessment, $C T$ computed tomography

Values of $p>0.05$ indicate no association between treatment and a given characteristic or factor and the 2 patients in group 3 who required intubation had been extubated and transferred to the regular inpatient ward.

Table 3 shows the laboratory data for the different treatment groups on admission, after 3 days, and after 1 month. On admission, there were no differences between treatment groups in the values of CRP, D-dimers, total lymphocytes, CD4, or CD8, except for the lower CD4 value on day 3 after admission in group 3.

To determine whether anakinra therapy was related to a better prognosis, the chi-squared statistical test of independence was used. In addition, the contingency coefficient was significant for the association between anakinra therapy and the patients' clinical course.

The 95\% confidence interval for the difference in the proportions of patients who died while on anakinra treatment and those who died in groups 1 and 2 indicated that the mortality rate was lowest in group 3 (0\% vs $11 \%, 95 \%$ CI [-0.164;-0.055]; $p=0.0354)$.

The Kaplan-Meier survival curves indicated a higher likelihood of survival at different time points in patients treated with anakinra (Figs. 1,2).

Cox regression models adjusted for the variables age, CURB-65 score and qSOFA score provided the risk ratio for anakinra treatment, which showed that the risk of death was reduced by almost $50 \%$ in patients who received this therapy. Anakinra was well tolerated in all patients, and no infectious complications were recorded.

\section{Discussion}

Our findings suggest that the use of anakinra in patients with moderate hyperinflammation associated with severe SARS-CoV-2 pneumonia after previous failure of corticosteroids alone or with tocilizumab therapy may be an alternative in the management of these patients, and may prevent deaths.

Immunosuppressive therapies are being used in patients with severe COVID-19 who develop hyperinflammatory state because of the pathogenic role of pro-inflammatory cytokines such as IL-1, IL-6 and TNF- $\alpha$. Corticosteroid therapy has been used initially and appears to reduce the mortality rate $[19,20]$. Preliminary data from the RECOVERY trial showed that in severe COVID-19 pneumonia requiring oxygen or mechanical ventilation, moderate doses of dexamethasone resulted in lower 28-day mortality [21]. Tocilizumab, a humanized monoclonal antibody against the human IL-6 receptor, has been proposed to reduce the risk of intubation and/or death [22-24] in patients with COVID19 pneumonia associated with hyperinflammatory state, although it should be noted that the follow-up period in these previous studies was less than 28 days. In the present study, the 60-day mortality rate was similar in the group of patients who received corticosteroids and the group that received corticosteroids plus tocilizumab. However, the percentage of patients who required intubation was higher in the latter group, a finding probably related to the fact that those patients with worse quality of life and lower life expectancy for whom tocilizumab was ruled out as an option were also not considered candidates for ICU admission. These observations suggest that in patients with hyperinflammatory state who do not respond to corticosteroids, the addition of tocilizumab may not reduce mortality. This is consistent with phase III results from the COVACTA trial [25], which did not meet its primary endpoint of improved clinical status in hospitalized patients with severe COVID-19-associated pneumonia or its secondary endpoint of patient mortality at week 4 . Together, these findings suggest the importance 
Table 3 Laboratory findings on admission, 3 days after admission, and 1 month after admission in different treatment groups

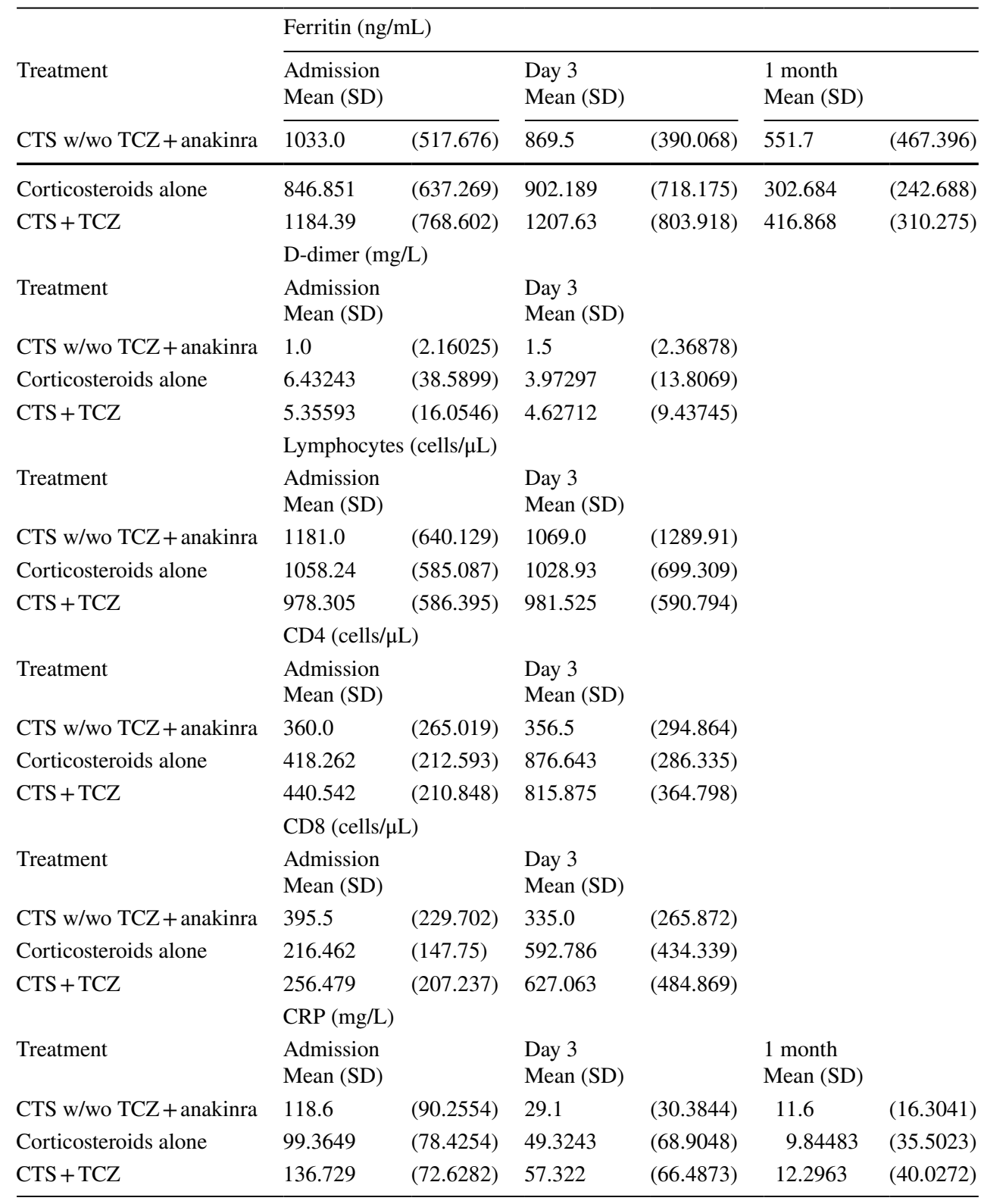

$S D$ standard deviation, $C T S$ corticosteroids, $T C Z$ tocilizumab, $C R P$ C-reactive protein of determining the specific inflammatory profile in patients who are candidates for tocilizumab [26].

Anakinra is an IL-1 receptor antagonist that may be an alternative biologic for patients suffering from severe COVID-19 pneumonia and hyperinflammation who fail previous treatment with corticosteroids alone or with tocilizumab. IL- $1 \alpha$ released by necrotizing lung cells may be one of the initial cytokines produced during Covid-19 pathogenesis. Upon binding to its receptor, this interleukin induces the synthesis of several cytokines such as IL- 6 , TNF- $\alpha$, granulocyte-macrophage colony-stimulating factor (GM-CSF), and IL-17. Another interleukin, IL-1 $\beta$, is mainly secreted by monocyte-macrophages to regulate cytokines, and also leads to the expression of tissue factors that contribute to hypercoagulability. In this context, the inhibition of IL-1 signaling with anakinra (an IL- $1 \alpha$ and IL- $1 \beta$ blocker) in patients with SARS-CoV-2 infection and hyperinflammation may block cytokine storm by acting upstream of the inflammatory signaling pathway [27]. This drug may also have an additional beneficial effect on the prothrombotic state in these patients, since IL-1 blockade has been shown to reduce mortality rates in patients with acute myocardial infarction, as well as in patients with severe sepsis, liver dysfunction, and disseminated intravascular coagulation [28-30].

In the present study, $95 \%$ of the patients presented associated comorbidities, although without differences between treatment groups on admission in their CURB-65 or qSOFA scores. The predictive factors associated with 
Fig. 1 Kaplan-Meier survival curve. Short vertical lines indicate patients alive but not discharged home, and considered as censored. Large steps indicate deaths ocurring during follow-up
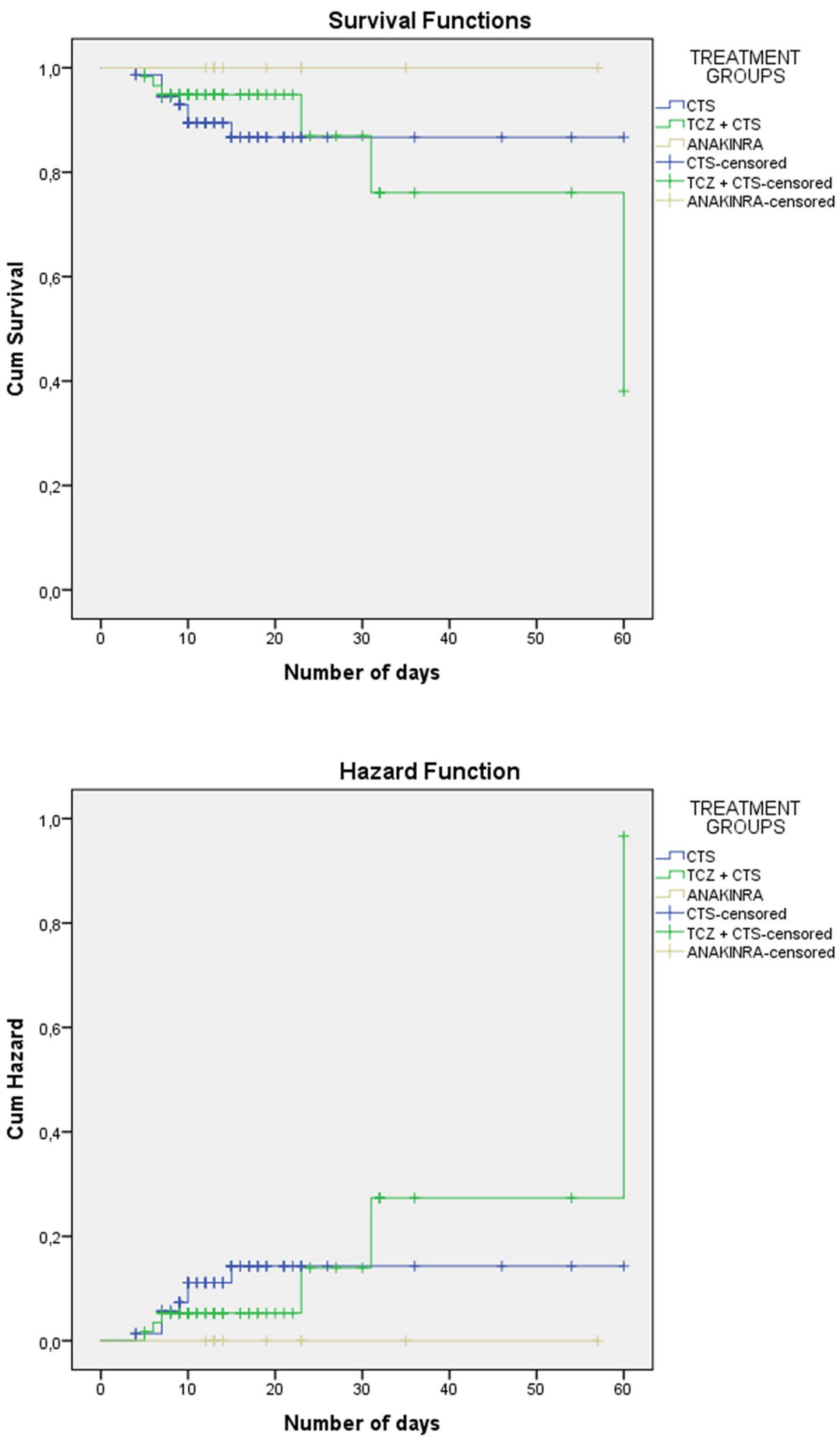

Fig. 2 Kaplan-Meier cumulative hazard curve. Short vertical lines indicate patients alive but not discharged home, and considered as censored. Larege steps indicate deaths ocurring during follow-up higher mortality rate and worse prognosis were age, diabetes mellitus, ischemic heart disease, obesity, heart failure, hypertension, and kidney failure. Older age was the most important factor in patients with SARS-CoV-2 infection and has been associated with the development of ARDS and subsequent death [31].

In the present study, the 30-day and 60-day mortality rate were lower in the group that received anakinra as a rescue 
treatment after failure to respond to corticosteroids alone or with tocilizumab. This may have been a result of using an early treatment strategy with anakinra in patients who had not improved or who had worsened within $48 \mathrm{~h}$ after receiving corticosteroids alone or with tocilizumab.

No differences were found in patients' clinical course (death or ICU admission) at 60 days between patients with multimorbidity or patients older than 65 years treated with corticosteroids (group 1) and those who received corticosteroids and tocilizumab (group 2).

Regarding the 10 patients who received anakinra (group 3) because their clinical status did not improve or worsened despite treatment with corticosteroids alone or with tocilizumab, their clinical course at 60 days (death or ICU admission) showed no association with previous treatment with corticosteroids alone or combined with tocilizumab. We postulate that IL-1 blockade may have acted synergistically with corticosteroids or IL-6 blockade, and thus led to additional beneficial effects in controlling the hyperinflammatory state.

The usefulness of anakinra therapy in patients with SARS-CoV-2 pneumonia who develop secondary hemophagocytic lymphohistiocytosis was recently reported [32]. This result together with the present findings suggest that anakinra could be administered instead of tocilizumab in patients with hyperinflammation associated with severe COVID-19 who have low serum IL-6 levels $(<40)$ after failed corticosteroid therapy. The different cytokine profiles in these patients may determine their clinical response to the blockade of specific cytokines, a consideration we believe is important in designing future studies.

The likely beneficial effects of anakinra on the common pattern of coagulopathy observed in patients with COVID19 were not associated with differences between groups in serum D-dimer levels before or after 3 days of treatment. Although IL-1 blockade has been associated with increased tissue factor expression, it is likely that other mechanisms derived from IL-1 blockade, such as reduced venous thromboinflammation and reduced platelet activation [33-35], may be beneficial in improving prothrombotic state in patients with severe SARS-CoV2 infection.

No differences were found in serum ferritin levels at 1 month between the three groups, suggesting that hyperinflammatory state was controlled to a similar degree in all patients regardless of their immunosuppressive treatment [36]. Although elevated ferritin levels have been considered a clinical factor associated with a poor prognosis in patients with cytokine storm [37], we observed no between-group differences in the association between serum ferritin levels and mortality - a finding probably related in part to the fact that the elevation in ferritin was moderate in all patients in the present cohort. Other laboratory factors associated with a worse prognosis, e.g. lymphopenia [38], were not associated with an increased mortality rate in any treatment group. The analysis of lymphocyte subpopulations showed that CD4 count was significantly lower on day 3 in the group treated with anakinra, although there were no significant differences between groups in CD8 count. Patients with severe respiratory failure associated with SARS-CoV-2 infection present CD4 depletion, so it is likely that IL-1 blockade contributes to an increase in circulating CD4 and thus helps to restore immune balance, with a better prognosis [39].

The dose of anakinra used in the present cohort was established based on pharmacokinetic criteria and was adjusted to body weight in an effort to minimize the risk of infection, given that our patients had previously received corticosteroids alone or with tocilizumab. It was decided to use an intermediate dosage of anakinra because we reasoned, based on our experience with anakinra in rheumatic and autoinflammatory diseases, that in patients with moderate hyperinflammation, this dosage would be effective in controlling inflammation without increasing adverse events. Huet et al. [13] showed that the use of lower dosages of anakinra, i.e. $100 \mathrm{mg}$ twice a day for $72 \mathrm{~h}$, then $100 \mathrm{mg}$ daily for 7 days, reduced both the need for invasive mechanical ventilation in the ICU and mortality among patients with severe forms of COVID-19, without serious side-effects. In one recently published study [40] the dose of anakinra associated with clinical improvement in patients with ARDS, hyperinflammation, and COVID-19 was higher $(10 \mathrm{mg} /$ $\mathrm{kg}$ /day); however, these patients had not been previously treated with corticosteroids and/or tocilizumab. This higher dose of anakinra was associated with a $24 \%$ rate of severe adverse effects and a $14 \%$ rate of infectious complications. In the present study, no patients had infectious complications, probably because of the lower dose of anakinra; this finding was also reported in a recent study [13] that used similar doses of anakinra.

The limitations of the present study are its retrospective observational design and the small sample size of the group that received anakinra. In addition, all the patients rescued with anakinra were male, a factor associated with greater severity, and this does not allow us to extrapolate our observations to women. In addition, we note that the sample size in group 3 (anakinra treatment) was small and that a larger sample size would likely influence the significance level. However, the patients were recruited consecutively, and the treatment protocol was the same for all patients. In addition, patients in all groups had similar clinical severity indexes and similar age distributions, making them a more or less homogeneous sample.

In conclusion, the use of anakinra in patients with moderate hyperinflammation associated with severe COVID-19 pneumonia after previous failure of corticosteroids alone or with tocilizumab therapy may be an alternative for the management of these patients and may reduce mortality. 
However, randomized clinical trials are needed to confirm the possible benefits of this therapeutic strategy.

Acknowledgements We thank all health care personnel for their outstanding work during this pandemic and $\mathrm{K}$. Shashok for improving the use of English in the manuscript.

Funding No funding was received.

\section{Compliance with ethical standards}

Conflict of interest The authors declare that they have no conflict of interest.

Statements on human and animal rights The study complies with Helsinki principles and declaration.

Informed consent Written informed consent were obtained from each patient, their legal representative or closest relative.

\section{References}

1. Lu H, Stratton CW, Tang YW (2020) Outbreak of pneumonia of unknown etiology in wuhan china: the mystery and the miracle. $\mathrm{J}$ Med Virol. https://doi.org/10.1002/jmv.25678

2. Zhou F, Yu T, Du R et al (2020) Clinical course ad risk factors for mortality of adult inpatients with COVID-19 in Wuhan China: a retrospective cohort study. Lancet. https://doi.org/10.1016/S0140 -6736(20)30566-3

3. Siddiqi HK, Mehra MR (2020) "COVID-19 illness in native and immunosuppressed states: a clinical-therapeutic staging proposal. J Heart Lung Transplant. https://doi.org/10.1016/j.healu n.2020.03.012

4. Wu C, Chen X, Cai Y et al (2019) Risk Factors Associated With Acute Respiratory Distress Syndrome and Death in Patients With Coronavirus Disease 2019 Pneumonia in Wuhan, China. JAMA Intern Med. https://doi.org/10.1001/jamainternmed.2020.0994

5. Ruan Q, Yang K, Wang W et al (2020) Clinical predictors of mortality due to COVID-19 based on an analysis of data of 150 patients from Wuhan, China. Intensive Care Med. https://doi. org/10.1007/s00134-020-05991

6. Mehta P, McAuley DF, Brown M et al (2020) COVID-19: consider cytokine storm syndromes and immunosuppression. Lancet. https://doi.org/10.1016/S0140-6736(20)30628-0

7. Ong EZ, Chan YFZ, Leong WY et al (2020) A Dynamic Immune Response Shapes COVID-19 Progression. Cell Host Microbe. https://doi.org/10.1016/j.chom.2020.03.021

8. Grimaldi D, Goicoechea Turcott EW, Taccone FS (2016) IL-1 Receptor antagonist in sepsis: new findings with old data? J Thorac Dis. https://doi.org/10.21037/jtd.2016.08.51

9. Cavalli G, Dinarello CA (2018) Anakinra therapy for non-cancer inflammatory diseases. Front Pharmacol. https://doi.org/10.3389/ fphar.2018.01157

10. Moteagudo LA, Boothby A, Gertner E (2020) Continuous intravenous anakinra infusion to calm the cytokine storm in macrophage activation syndrome. ACR Open Rheumatol. https:// doi.org/10.1002/acr2.11135

11. Navarro-Millán I, Sattui SE, Lakhanpal A et al (2020) Use of anakinra to prevent mechanical ventilation in severe COVID
-19: a case series. Arthritis Rheumatol. https://doi.org/10.1002/ art. 41422

12. Pontali E, Volpi S, Antonucci G et al (2020) Safety and efficacy of early high-dose IV anakinra in severe covid-19 lung disease. J Allergy Clin Inmunol. https://doi.org/10.1016/j. jaci.2020.05.002

13. Huet T, Beaussier H, Voisin O et al (2020) Anakinra for severe forms of CoVid-19: a cohort study. Lancet Rheumatol. https://doi. org/10.1016/S2665-9913(20)30164-8

14. Langer-Gould A, Smith JB, Gonzales EF et al (2020) Early identification of COVID-19 cytokine storm and treatment with anakinra or tocilizumab. Int J Infect Dis. https://doi.org/10.1016/j. ijid.2020.07.081

15. Prokop M, van Everdingen W, van Rees VT et al (2020) CORADS- A categorical CT assessment scheme for patients with suspected COVID-19: definition and evaluation. Radiology. https ://doi.org/10.1148/radiol.2020201473

16. Lim WS, Van der Eerden MM, Laing R et al (2003) Defining community acquired pneumonia severity on presentation to hospital: an international derivation and validation study. Thorax. https:// doi.org/10.1136/thorax.58.5.377

17. Jiang J, Yang J, Jin Y et al (2018) Role of qSOFA in predicting mortality of pneumonia: a systematic review and meta-analysis. Medicine (Baltimore). https://doi.org/10.1097/MD.0000000000 012634

18. COVID-19: BSTI STATEMENT AND GUIDANCE: https://www. bsti.org.uk/standards-clinicalguidelines/clinical-guidelines/covid -19-bsti-statement-and-guidance/INTERNET. Accessed 15 March 2020.

19. Fadel R, Morrison AR, Vahia A et al (2020) Early short course corticosteroids in hospitalized patients with COVID-19. Clin Infect Dis. https://doi.org/10.1093/cid/ciaa601

20. Callejas Rubio JL, Luna del Castillo JD, De la Hera Fernández $\mathrm{J}$ et al (2020) Effectiveness of corticoid pulses in patients with cytokine storm syndrome induced by SARS-CoV-2 infection. Med Clin. https://doi.org/10.1016/j.medclin.2020.04.018

21. Horby P, Lim WS, Emberson JR, and the Members of the RECOVERY Collaborative Group (2020) Dexamethasone in hospitalized patients with Covid-19 preliminary report. NEJM. https://doi.org/10.1056/NEJMoa2021436

22. Guaraldi G, Meschiari M, Cozzi-Lepri A et al (2020) Tocilizumab in patients with severe COVID-19: a retrospective cohort study. Lancet Rheumatol. https://doi.org/10.1016/S2665-9913(20)30173 $-9$

23. Somers EC, Eschenauer GA, Troost JP et al (2020) Tocilizumab for treatment of mechanically ventilated patients with COVID-19. Clin Infect Dis. https://doi.org/10.1093/cid/ciaa954

24. Capra R, De Rossi N, Mattioli F. Impact of low dose tocilizumab on mortality rate in patients with COVID-19 related pneumonia Eur J Intern Med. 2020. doi: https://doi.org/10.1016/j. ejim.2020.05.009.

25. A Study to Evaluate the Safety and Efficacy of Tocilizumab in Patients With Severe COVID-19 Pneumonia (COVACTA). Phase III trial NCT number: NCT04320615

26. van Kraaij TD, Mostard RL, Ramiro S et al (2020) Tocilizumab in severe COVID-19 pneumonia and concomitant cytokine release syndrome. Eur J Case Rep Intern Med. https://doi.org/10.12890 12020_001675

27. Brucato A, Emmi G, Cantarini L et al (2018) Management of idiopathic recurrent pericarditis in adults and in children: a role for IL-1 receptor antagonism. Intern Emerg Med 13(4):475-489. https://doi.org/10.1007/s11739-018-1842-x

28. Ridker PM, Libby P, MacFadyen JG et al (2018) Modulation of the interleukin- 6 signaling pathway and incidence rates of atherosclerotic events and all-cause mortality: analyses from the 
Canakinumab Anti-Inflammatory Thrombosis Outcomes Study (CANTOS). Eur Heart J. https://doi.org/10.1093/eurheartj/ehy310

29. Shakoory B, Carcillo JA, Chatham W et al (2016) Interleukin -1 receptor blockade is associated with reduced mortality in sepsis patients with features of macrophage activation syndrome: reanalysis of a prior phase III trial. Crit Care Med. https://doi. org/10.1097/CCM.0000000000001402

30. Yadav V, Chi L, Zhao R et al (2019) ENTPD-1 disrupt inflammasome IL-1beta-driven venous thrombosis. J Clin Invest. https:// doi.org/10.1172/JCI124804

31. Chaomin Wu, Xiaoyan C, Yanping C et al (2019) Risk factors associated with acute respiratory distress syndrome and death in patients with coronavirus disease 2019 pneumonia in Wuhan, China. JAMA Intern Med. https://doi.org/10.1001/jamaintern me.2020.0994

32. Dimopoulos G, de Mast Q, Markou N et al (2020) Favorable anakinra responses in severe Covid19 patients with secondary hemophagocytic lymphohistiocytosis. Cell Host Microbe. https ://doi.org/10.1016/j.chom.2020.05.007

33. Gupta N, Sahu A, Prabhakar A et al (2017) Activation of NLRP3 inflammasome complex potentiates venous thrombosis in response to hypoxia. Proc Natl Acad Sci USA. https://doi.org/10.1073/ pnas. 1620458114

34. Karmakar M, Katsnelson M, Malak HA et al. Neutrophil IL-1betta processing induced by pneumolysin is mediated by theNLRP3/ ASC inflammasome and caspase- 1 activation and is dependent on K+ efflux J Immunol 2015. doi: https://doi.org/10.4049/jimmu nol.1401624

35. Saxena A, Chen W, Su Y et al (2013) IL-1 induced proinflammatory leukocyte infiltration and regulated fibroblast phenotype in the infarcted myocardium. J Immunol. https://doi.org/10.4049/ jimmunol.1300725

36. Furong Zeng F, Huang Y, Guo Y et al (2020) Association of inflammatory markers with the severity of COVID-19: A metaanalysis. Int J Infect Dis. https://doi.org/10.1016/j.ijid.2020.05.055

37. Michael Henry B, Santos de Oliveira MH, Benoit S, Plebani M, Lippi G (2019) Hematologic, biochemical and immune biomarker abnormalities associated with severe illness and mortality in coronavirus disease 2019 (COVID-19): a meta-analysis. Clin Chem Lab Med. https://doi.org/10.1515/cclm-2020-0369

38. Wang F, Nie J, Wang H et al (2020) Characteristics of Peripheral Lymphocyte Subset Alteration in COVID-19 Pneumonia. J Infect Dis. https://doi.org/10.1093/infdis/jiaa150

39. Giamarellos-Bourboulis EJ, Netea MG, Rovina N et al (2020) Complex immune dysregulation in COVID-19 patients with severe respiratory failure. Cell Host Microbe. https://doi. org/10.1016/j.chom.2020.04.009

40. Cavalli G, De Luca G, Campochiaro C et al (2020) Interleukin-1 blockade with high-dose Anakinra in patients with COVID19, acute respiratory distress syndrome, and hyperinflammation: a retrospective cohort study. Lancet Rheumatol. https://doi. org/10.1016/S2665-9913(20)30127-2

Publisher's Note Springer Nature remains neutral with regard to jurisdictional claims in published maps and institutional affiliations. 